Document downloaded from:

http://hdl.handle.net/10251/67458

This paper must be cited as:

Olguín Pinatti, CA.; Laguarda-Miro, N.; Garcia-Breijo, E.; Martínez-Máñez, R.; Soto Camino, J. (2014). An electronic nose for the detection of Sarin, Soman and Tabun mimics and interfering agents. Sensors and Actuators B: Chemical. 202:31-37. doi:10.1016/j.snb.2014.05.060.

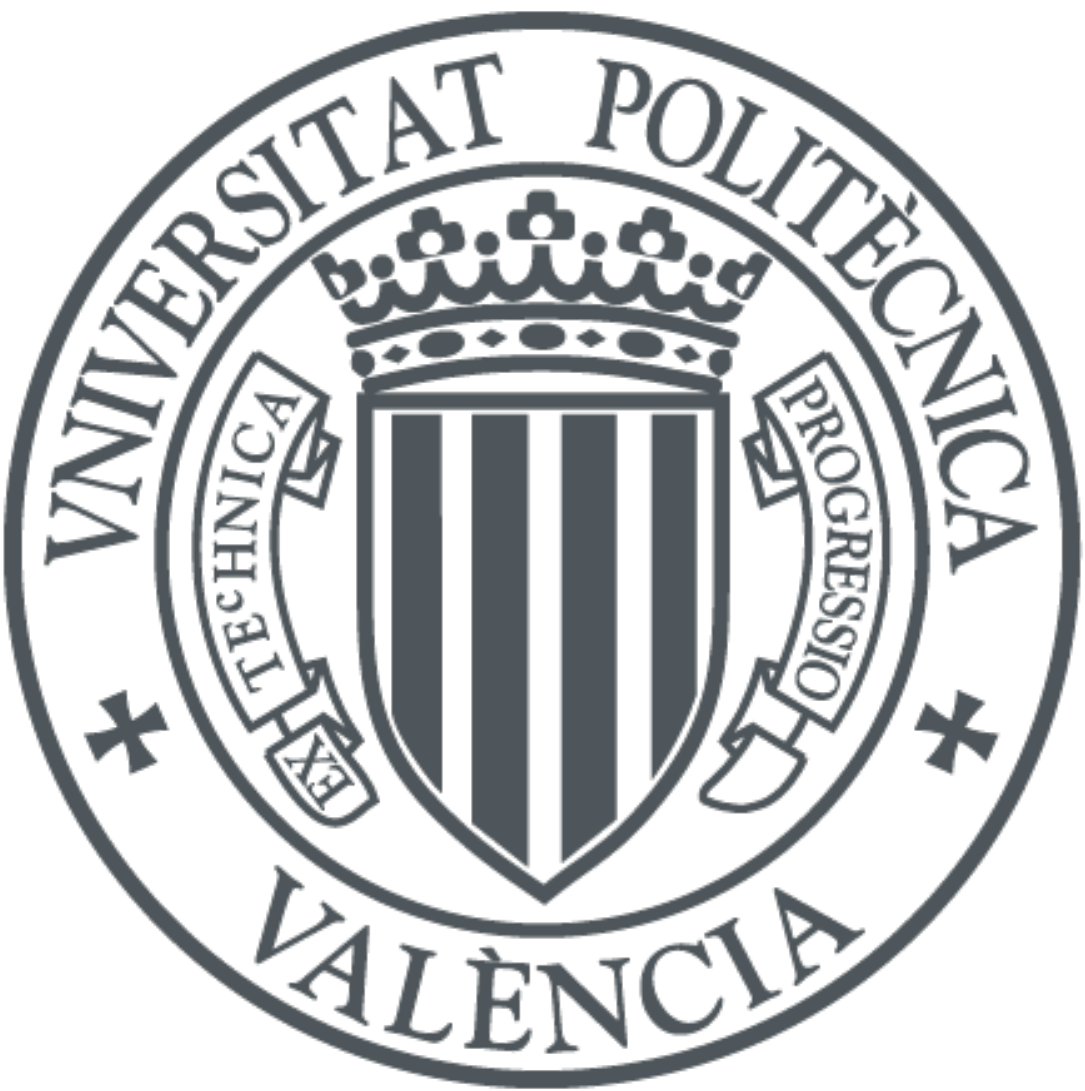

The final publication is available at

http://dx.doi.org/10.1016/j.snb.2014.05.060

Copyright Elsevier

Additional Information 


\title{
An Electronic Nose for the detection of Sarin, Soman and Tabun mimics and interfering agents.
}

\author{
Cristian Olguín, ${ }^{1}$ Nicolás Laguarda-Miró, ${ }^{1,3}$ Lluís Pascual, ${ }^{1,2}$ Eduardo García-Breijo, ${ }^{1,5}$ Ramón \\ Martínez-Mañez, ${ }^{1,2,4}$ Juan Soto, ${ }^{1,2}$ \\ 1 Centro de Reconocimiento Molecular y Desarrollo Tecnológico (IDM), Unidad Mixta Universidad Politécnica de Valencia - \\ Universidad de Valencia, Valencia, Spain. \\ 2 Departamento de Química. Universidad Politécnica de Valencia. Camino de Vera s/n. E-46022, Valencia, Spain. \\ 3 Departamento de Ingeniería Química y Nuclear. Universidad Politécnica de Valencia. Camino de Vera s/n. E-46022, Valencia, \\ Spain. \\ 4 CIBER de Bioingeniería, Biomateriales y Nanomedicina (CIBER-BBN). \\ 5 Departamento de Electrónica. Universidad Politécnica de Valencia. Camino de Vera s/n. E-46022, Valencia, Spain.
}

\section{Abstract}

An electronic nose system (E-nose) with metal oxide semiconductor sensors (MOS) has been designed to discriminate and quantify different chemical warfare agents (CWA) mimics. The Enose consists of an array of commercial MOS sensors for different gases, two sensors for temperature sensing, a sample handling system, a data acquisition system and a laptop with the data acquisition system control. With this device, discrimination studies have been carried out to detect specific CWA simulants (diethyl chlorophosphonate (DCP), diethyl cyanophosphate (DCNP), diisipropyl fluoride (DFP)), their derivatives (diethyl 1-phenylethyl phosphonate (OP-1), diethyl (2- cyanoethyl)phosphonate (OP-2), dimethyl methyl phosphonate (OP-3) and diethyl (2-oxopropyl)phosphonate (OP-4)) and some potential interfering substances (sulfuric acid, ammonia, ethanol and acetone). Principal Components Analyses (PCA) show that it is possible to discriminate the studied organophosphorous CWA mimics (DCP, DCNP and DFP) from the other studied derivatives and potential interfering agents. In addition, DCNP quantification studies have been done by using Partial Least Squares (PLS) and a mathematical model has been obtained to predict DCNP concentrations in air. In this model, the coefficient of determination $\left(R^{2}\right)$ is 0.9567 , the root mean square error of prediction (RMSEP) is 30 and the limit of detection (LOD) is $5 \mathrm{ppm}$ so the model is considered valid. These results suggest that this E-nose system is capable to discriminate and quantify CWA mimics and it would be a feasible system to be used in a real scenario. 
The term "Electronic Nose" was first used in 1988 by Gardner and Bartlett, who defined it as "an instrument which comprises an array of electronic chemical sensors with partial specificity and appropriate pattern recognition system, capable of recognizing simple or complex odours" $[1,2]$. Due to the characteristic response pattern provided by the array of unspecific sensors, this Electronic Nose System is capable to give information about the surrounding environment. So, it is possible to identify and quantify certain gaseous compounds using an appropriate data analysis technique.

The first electronic nose model was provided by Dodd and Persaud. Their system was based on three different metal oxide sensors and it was able to identify several gases by using the measured steady-state signals of these three sensors [3]. Nowadays, Electronic Noses have evolved considerably and there are several technologies that can be applied in these devices such as surface acoustic wave [4], metal oxide semiconductor field effect transistors [5], conducting polymers [6], optical sensors [7], gas chromatography [8], ion mobility spectroscopy [9], infrared spectroscopy [10], etc. [11, 12].

Due to the Biological and Toxic Weapons Conventions on the prohibition of the development, production and stockpiling of bacteriological and toxin weapons and on their destruction signed at London, Moscow and Washington on 10 April 1972 and revised in 1993, chemical warfare agents (CWA) shouldn't be in use. However nowadays, the chemical warfare is still a real problem. The threat of exposure to chemical warfare agents has been considered a military issue. However, several recent events have demonstrated that civilians may also be exposed to these agents. Chemical warfare agents are defined as "chemical substances, gaseous, liquid or solid, which might injure humans or animals" [13, 14]. Chemical warfare agents are extremely toxic and have severe effects on human and animal health, either as a gas or liquid and poisoning may occur by gas inhalation, contact with skin or polluted liquid/food consumption.

Our investigation reported herein focuses on G-type organophosphorous nerve agents as Sarin(GB), Soman(GD) and Tabun(GA) whose effects in the organism are due to their ability to inhibit the action of acetylcholinesterase [16]. Given the high toxicity of nerve gases, organophosphorous model compounds, such as diethyl chlorophosphonate (DCP), diethyl cyanophosphate (DCNP), diisipropyl fluoride (DFP), which has a similar structure and reactivity as nerve agents but display less toxicity, are generally used in studies in the laboratory [34, 35]. The close reactivity is related with the presence of "similar" leaving groups (i.e. $\mathrm{F}, \mathrm{Cl}$ and $\mathrm{CN}$ ) in 
DFP, DCP and DCNP to those found in Sarin, Soman and Tabun (i.e. F and CN). Moreover DFP, DCP and DCNP are less toxic and in fact are not viable nerve agents because are readily hydrolysed (poorly persistent) when compared with Sarin, Soman and Tabun. The mimics used in the laboratory were also organophosphorous and organophosphate compounds such as diisipropyl fluoride (DFP), diethyl chlorophosphonate (DCP), diethyl cyanophosphate (DCNP).

Nowadays, there are several equipments and analytical methods that have been approved by the Chemical Weapons Convention in 1993 for chemical warfare agents in-situ detection and quantification [16, 17]. Air monitoring systems for nerve agents are mainly based on ion mobility spectroscopy (IMS) or gas chromatography coupled with mass spectrometry (GC/MS). However, these systems commonly present several difficulties, for instance: Analyses have to be carried out in a laboratory, qualified personnel is required to operate these devices, instrumental and chemicals are complex, the equipment is expensive and the analyses are time consuming. Due to these disadvantages, some alternative methods have been rising such as surface acoustic wave devices [18], electrochemistry [19], spectrophotometric sensors [20], immunochemical sensors [21], capillary electrophoresis [22], enzymatic assays [23], chromofluorogenic probes [24, 25], chemiresistive sensors [26] and liquid crystals [27].

\section{Theory}

\subsection{Principle of operation}

Despite MOS sensors have problems with humidity (as water is an interfering compound for this kind of sensors) we have used them in our system because MOS sensors are robust, common and easy to buy everywhere. Therefore, in order to compensate the potential interference of water, a humidity sensor has been included in the system. On the other hand, as temperature is another potential interfering factor, two temperature sensors have also been included in the system. A detailed explanation of the MOS sensors operation is provided elsewhere [28].

\subsubsection{Chemical Principle}

MOS sensors use metal oxide-based sensing thick films deposited onto a silica substrate. The substrate contains electrodes that measure the resistance of the sensing layer and a heater to desorb any volatile compound remaining in sensing layer by increasing the temperature of the sensor. The sensing layer is a porous thick film made of polycrystalline $\mathrm{SnO}_{2}$. So the gases to be measured are adsorbed in this surface. 
In a clean atmosphere, both oxygen and water vapor-related species are adsorbed on the surface of the $\mathrm{SnO}_{2}$ grains but, when other pollutant gasses are present, a series of reactions take place in the sensor's surface. In case of having reducing gases such as $\mathrm{CO}$ or $\mathrm{H}_{2}$, a reaction takes place with the pre-adsorbed oxygen and water vapor-related species which decreases the resistance of the sensor. Instead, when oxidizing gases such as $\mathrm{NO}_{2}$ and $\mathrm{O}_{3}$ are present, the resistance increases. The magnitude of the changes depends on the microstructure and the composition/doping of the base material, on the morphology and the geometrical characteristics of the sensing layer and substrate, as well as on the temperature at which the sensing takes place [28].

In order to explain the resistance change in the sensor when measuring nerve agent mimics the reaction mechanisms for DMMP sample is shown [29], (these mechanisms can be extrapolated to other components and they help the understanding of how the MOS sensors work). DMMP is quite thermally stable at temperatures between $300^{\circ} \mathrm{C}$ and $600^{\circ} \mathrm{C}$. Its degradation generates two compounds: carbon dioxide and methylphosphonic acid as shown in Figure 1.

[Insert Figure 1]

The decrease in resistance obtained when DMMP is detected, takes place in two stages. First, DMMP is adsorbed onto the $\mathrm{SnO}_{2}$ surface, allowing it to react with an oxygen species $\left(\mathrm{O}^{-}\right)$. This reaction leads to the formation of methylphosphonic acid, which remains adsorbed onto the $\mathrm{SnO}_{2}, \mathrm{CO}_{2}$ which does not react with the sensor, and $\mathrm{H}_{2} \mathrm{O}$. At the same time, electrons can be released to the conduction band leading to a decrease in the $\mathrm{SnO}_{2}$ resistance as shown in Figure 2.

\section{[Insert Figure 2]}

Finally, the last stage of this mechanism leads to the formation of an ionic phosphorous compound, which is adsorbed onto the $\mathrm{SnO}_{2}$ (Figure 3).

$$
\text { [Insert Figure 3] }
$$

\subsubsection{Transducer Principle}

Composition changes in the environment will determine changes in resistance on the sensing layers. The relationship between sensor resistance and the concentration of the target gas (the gas or gases to which the sensor is designed) usually follows a power law described below: 
Where ' $c$ ' is the concentration of the target gas, ' $\mathrm{K}$ ' is a measurement constant and ' $b$ ' is a value in the range [0.3-0.8]. The positive sign is used for oxidizing gases and the negative sign for the reducing ones.

133

134

135

136

137

\section{Materials and methods}

\subsection{Chemicals}

All the chemical compounds have been purchased from Sigma-Aldrich. Discrimination studies have been carried out at their respective saturated vapour concentrations (see Table 1). In addition, quantification studies have been developed in the range [0-208(sat)] ppm.

\subsection{Experimental}

In this paper, two types of experiments have been done: First, a study has been carried out to discriminate among the different mimic agent samples and some potential interfering substances. Secondly, a quantification study of one of the mimics has been done in order to be able to assess the concentration of this compound in a sample and obtain the limit of detection (LOD).

First of all, discrimination studies have been carried out by using the selected nerve agent simulants (diethyl chlorophosphonate (DCP), diethyl cyanophosphate (DCNP), diisipropyl fluoride (DFP)), and a set of four similar organophosphorous derivatives (diethyl 1-phenylethyl phosphonate (OP-1), diethyl (2- cyanoethyl)phosphonate (OP-2), dimethyl methyl phosphonate (OP-3), and diethyl (2-oxopropyl)phosphonate (OP-4)). On the other hand, a set of different potential interfering samples were studied: Sulfuric acid and ammonia samples were prepared and measured to analyze the system's response when acid and basic vapors are present. The influence of ethanol and acetone was also studied using them as a reference of the system's response to the presence of volatile solvents. All of them have been measured independently using the same controlled environment and assay specifications in order to avoid the effect of external factors.

[Insert Table 1]

The samples shown in Table 1 were measured under real conditions in order to study the strength of the system. The evaporation chamber was the only part of the device that was under control. In this way, it is possible to ensure that changes in the response signal are only 
due to changes in the measured sample. The gas to be measured has been mixed with normal air and not with a pattern gas. Next, measurements of the samples were carried out in different seasons throughout the year in order to develop assays in a wide range of ambiance conditions. All the samples were measured randomly including the repetitions of each sample as all of them were analyzed by triplicate. Consequently, all the analyses were carried out completely random.

Quantification studies have been carried out using DCNP due to its higher response and selectivity observed in discrimination studies previously conducted. A simple dosage system was used to introduce different known concentrations of DCNP into the system (Table 2).

DCNP $(500 \mu \mathrm{L})$ were deposited and evaporated in a $500 \mathrm{~mL}$ thermostated balloon at $25^{\circ} \mathrm{C}$ and vacuum until gas saturation was reached. Then, a controlled volume was extracted with a syringe and injected into the measurement chamber. First, an equal volume of air must be extracted from the measurement chamber before the sample injection in order to avoid overpressure. The injected volumes are those shown in Table 2.

[Insert Table 2]

\subsection{Equipment}

The equipment has been designed, developed and manufactured by the Group of Electronic Development and Printed Sensors member of the Center of Molecular Recognition and Technological Development (IDM) at the Polytechnic University of Valencia (UPV) and it was previously used to detect maturation on fruit [30].

The equipment (named E-nose system) consists of an array of commercial MOS sensors (FIGARO Engineering Inc., Japan) for different gases (hydrogen, carbon monoxide, butane, methane, etc.), two LM35DZ for temperature sensing, a sample handling system, a data acquisition system and a laptop with the data acquisition system control. The complete E-Nose System is shown in Figure 4. 
This E-nose is capable to handle an array of 15 sensors. One of the advantages of the system is that all the sensors can be configured independently (even different to those specified by the manufacturer). So, there are several possible configurations for each sensor. The idea is to use the nonspecific behavior of the sensor to recognize patterns. Therefore, the system has been designed to be flexible and use the sensors as a complex array and not exclusively to detect their corresponding specific gas.

Concerning the used sensors, Table 3 shows the list of the specific sensors in the E-Nose.

$$
\text { [Insert Table 3] }
$$

\subsubsection{Sample handling system}

The sample handling system includes two chambers: the concentration chamber (where samples are placed) and the measurement chamber (where the sensors array is placed). The concentration chamber has a cylindrical shape $(12 \mathrm{~cm}$ i.d $\times 16 \mathrm{~cm} \mathrm{~h}$.) and is connected to the measurement chamber $(12 \mathrm{~cm}$ i.d $\times 14 \mathrm{~cm} \mathrm{~h}$.) through a BTC diaphragm pump (Brushless Motor model H054B-11 from Hargraves) especially designed for gas flow and it has a diaphragm that is compatible with this type of dangerous gasses. The sample handling system also includes two stopcocks to control the gas flow. In this way, the sample handling system is flexible in configuration.

When a measurement has finished, the heating process ensures desorption of all the remaining molecules in the sensors. Vacuum is also applied to the system in order to assure the removal of every volatile compound from the sample handling system. In this way, the sensors become ready to be used again.

\subsubsection{Data acquisition system}

The data acquisition system includes the control for each sensor and the measuring electronic system. It has a master-slave structure. All slave boards are controlled by the master board that gather the data of the 15 slaves and send them to the PC. Each slave controls several parameters of the sensor such as the supply voltage $\left(V_{C}\right)$, heating voltage $\left(V_{H}\right)$, load resistance $\left(R_{L}\right)$ and polarization pulses. These parameters can be configured through the PC by using an own software. The slave is based on a PIC18F2580 microcontroller, a 12-bits analogical-digital converter (AD7237A) and a 10-bits digital potentiometer (MAX5481). The master is based on a 
PIC18F4550 microcontroller that controls the communication between the PC and the slaves;

220 furthermore it controls the whole gas flow system.

\subsubsection{Data acquisition system control}

In order to handle the entire system, a software interface has been designed. This software allows the user to configure the sensors and control the parameters of the experiment. The parameters are configured by the user via software and they are sent to the master through a serial port. The master sends the configuration data to every slave by using an $I^{2} \mathrm{C}$ bus. Next, the slave-microcontroller configures the digital-analogical converter (DAC) to supply $V_{C}$ and $V_{H}$ to the sensor. If pulses are required, the slave-microcontroller also configures these voltages temporarily. Then, the microcontroller modifies the value of $R_{L}$ through the digital potentiometer by a serial peripheral interface (SPI) protocol. The implemented software has three main parts.

The first one is the data acquisition control application, in which we can control the parameters of every assay such as the time of the probe, the cleaning process, the diagnosis test, etc.

The second part of the implemented software is a display showing the result of the measuring.

The last part of the software is the sensors configuration application. It makes the system versatile and let the user configure all sensors separately and control the number of sensors involved in our system. In addition, this application let the user define all the operating point parameters: heater supply voltages, sensor supply voltages, heater heating/cooling times, sensor connection/disconnection times, measurement time, test establishment time, as well as the assignation of the sockets to the sensors. In fact, this important advantage let the user chose among different configurations. Moreover, it supplies information of the manufacturer about the nominal performance and security values of the different sensors.

\section{Results and discussion}

As a preliminary way to detect and discriminate nerve agent mimics and interfering substances, a principal components analysis (PCA) has been done with the obtained data from the studied samples. Next, an experiment to predict the concentration of DCNP was performed by using the partial least square technique (PLS). All statistical analyses were performed using the Solo (version 7.0.3, Eigenvector Research, Inc) software application. 
250 As PCA is an efficient approach to show a dataset in two dimensions, principal component 1

251 (PC1) and principal component 2 (PC2), with the maximum representativity, the responses of 252 different organophosphorous nerve agents simulants were analysed by this linear 253 unsupervised method. In addition, a set of potential environmental interferents such as solvents, acid and basic compounds was also analysed in order to determine the hardiness of our system in a non-ideal environment.

256 Figure 5 shows a PCA analysis developed using data from all the measured samples. This PCA is 257 an approach of how the system might work in real conditions. It can be seen that there is an 258 effective discrimination among types of samples.

[Insert Figure 5]

Figure 6 shows a second PCA model developed including only organophosphorous compounds in order to analyse the response of the system just with chemically similar samples. As shown, there is a clear discrimination among types of samples.

According to the obtained results our system is able to discriminate well among DCP, DFP, DCNP, typical organophosphorous interfering agents with similar structure, and some potential environmental interfering agents. Principally, DCNP is the easiest discriminated compound as DCP and DFP are also easily detected but their discriminations are not as selective as DCNP's discrimination is.

\subsection{PLS Quantification}

A quantification study using PLS was carried out $[31,32,33]$ in order to evaluate the performance of the system and determine the LOD for nerve agents simulants. According to the obtained results in previous classification studies, DCNP was selected as quantification analyte due to its high response.

The data collected was divided into two subsets; the first one was used to calibrate the model and the second one to test it with independent data. The Leave-One-Out approach has been used as cross-validation method, just using the training samples. According to the crossvalidation variance studies, 6 latent variables have been used to build the model. In order to 
281

282

283

284

285

286

287

288

289

290

291

292

293

294

295

296

297

298

299

300

301

302

303

304

305

306

307

308

309

ppm and then analysed by PLS. The calibration of the model was performed using 22 samples so the remaining 11 samples were used to test the model. Figure 7 shows the predicted values versus the real ones for DCNP. In this model, the coefficient of determination $\left(R^{2}\right)$ is 0.9567 and the root mean square error of prediction (RMSEP) is 30 so the model is considered statistically valid. In addition, the estimated LOD is $5 \mathrm{ppm}$. These values let us affirm that it is feasible to quantify warfare gas mimics by combining an electronic nose and this kind of mathematical models.

[Insert Figure 7]

\section{Conclusions}

A new method for nerve agents' mimics detection is introduced using a new device (E-Nose). Classification studies by PCA analyses show that the E-Nose system is able to discriminate the mimics of the main G-type nerve agents (DCP, DFP and DCNP) from typical organophosphorous derivatives and some potential interfering compounds such as acids, bases and solvents. These assays reveal that DCNP is the compound that shows a higher response. So, it was selected to carry out quantification studies. These determinations were performed by using PLS analyses and they showed statistically valid models. For the best of the obtained models, the coefficient of determination is 0.9567, RMSEP is 30 and the LOD for DCNP is 5 ppm.

Finally, according to these preliminary obtained results, the introduced E-Nose seems to be a reliable system to detect and quantify nerve agent mimics in complex samples with specific potential interfering substances. This system provides a selective and statistically valid response, in short measurement times; it is easy to use and cheap. These results give rise to begin the development of specific easy to use equipment for early detection of CWA's.

\section{Acknowledgements}

Financial support from the Spanish Government (MAT2009-14564-C04-02and MAT200914564-C04-01), the grant BES-2010-034031 from the FPI-MINECO Subprogram and the Spanish Government and European FEDER funds (MAT2012-38429-C04-04) are gratefully acknowledged.

\section{References}


310 [1] J. W. Gardner, P. N. Bartlett, A brief history of electronic noses, Sensors and Actuators B 18-19 (1994) 211-220.

312 [2] J. W. Gardner, P. N. Bartlett, Electronic Noses. Principles and Applications, Oxford 313 University, 1999.

314 [3] K. C. Persaud, G. Dodd, Analysis of discrimination mechanism in the mammalian 315 olfactory system using a model nose, Nature 299 (1982) 352-355.

316 [4] N. Barié, M. Bücking, M. Rapp, A novel electronic nose based on miniaturized SAW 317 sensor arrays coupled with SPME enhanced headspace-analysis and its use for rapid 318 determination of volatile organic compounds in food quality monitoring, Sensors and 319 Actuators B 114 (2006) 482-488.

320 [5] E. L. Kalman, A. Löfvendahl, F. Winquist, I. Lundström, Classification of complex gas 321 mixtures from automotive leather using an electronic nose, Analytica Chimica Acta 403 (2000) $32231-38$.

323 [6] A. Guadarrama, M.L. Rodríguez-Méndez, C. Sanz,, J.L. Ríos, J.A. de Saja, Electronic nose 324 based on conducting polymers for the quality control of the olive oil aroma. Discrimination of 325 quality, variety of olive and geographic origin, Analytica Chimica Acta 432 (2001) 283-292.

326 [7] C. Di Natale, D. Salimbeni, R. Paolesse, A. Macagnano, A. D’Amico, Porphyrins-based 327 opto-electronic nose for volatile compounds detection, Sensors and Actuators B 65 (2000) $328 \quad 220-226$.

329 [8] J. Olssona, T. Börjesson, T. Lundstedt, J. Schnürer, Volatiles for mycological quality 330 grading of barley grains: determinations using gas chromatography-mass spectrometry and 331 electronic nose, International Journal of Food Microbiology 59 (2000) 167-178.

332 [9] W. Vautz, J. I. Baumbach, J. Jung, Beer Fermentation Control Using Ion Mobility 333 Spectrometry - Results of a Pilot Study, Journal of the Institute of Brewing 112-2 (2006) 157334164

335 [10] D. Cozzolino, H. E. Smytha, K. A. Lattey, W. Cynkar, L. Janik, R. G. Dambergs, I. L. Francis, 336 M. Gishen, Combining mass spectrometry based electronic nose, visible-near infrared 337 spectroscopy and chemometrics to assess the sensory properties of Australian Riesling wines, 338 Analytica Chimica Acta 563 (2006) 319-324. 

Chem. Rev. 108 (2008) 705-725.

[12] R. D. S. Yadava, R. Chaudhary, Solvation, transduction and independent component analysis for pattern recognition in SAW electronic nose, Sensors and Actuators B 113 (2006) 121.

344 [13] Chemical Warfare Agents: An Overview of Chemicals defined as Chemical Weapons. 345 Organization for the Prohibition of Chemical Weapons, April 29, 1997, Assessed 11/20/2001.

346 [14] T. Wingfield, Military Law Review 1999, 162, 180; M. M. Kaplan, Bulettin of the World 347 Health Organization 1999, 77, 149; K. Sugendran, P. Kumar, R. Vijayaraghavan, Defense Sci. J. 1998, 48, 155.

349 [15] I. Campos, L. Gil, R. Martínez-Máñez, J. Soto, J. Luis Vivancos, Use of a Voltammetric 350 Electronic Tongue for Detection and Classification of Nerve Agent Mimics, Electroanalysis 2214 (2010) 1643-1649.

[16] S. M. Somani, R. P. Solana, S. N. Dube, Toxicodynamics of nerve agents, Chemical warfare agents 68 (1992).

354 [17] H. H. Hill, S. J. Martin, Conventional analytical methods for chemical warfare agents, Pure Appl. Chem 74-12 (2002) 2281-2291.

356 [18] A. T. Nimal, U. Mittal, M. Singh, M. Khaneja, G. K. Kannan, J. C. Kapoor, Development 357 of handheld SAW vapor sensors for explosives and CW agents, Sensors and Actuators B 135 358 (2009) 399-410.

359 [19] M. H. Hammond, K. J. Johson, S. L. Rose-Pehrsson, J.Ziegler, H. Walker, K. Coudy, D. 360 Gary, D. Tillett, A novel chemical detector using cermet sensors and pattern recognition 361 methods for toxic industrial chemicals, Sensors and Actuators B 116 (2006) 135-144.

362 [20] A. L. Jenkins, O. M. Uy, G. M. Murray, Polymer-based lanthanide luminescent sensor 363 for detection of the hydrolysis product of the nerve agent Soman in water, Anal. Chem. 71 364 (1999) 373-378.

365 [21] D. E. Lenz, A. A. Brimfield, L. A. Cook., Development of immunoassays for detection of 366 chemical warfare agents, in: D. A. Aga and E. M. Thurman (Eds.), Immunochemical Technology 367 for Environmental Applications, American Chemical Society, Washington, 1997, pp. 77-86.

368 [22] C. E. Kientz, E. W. J. Hooijschuur, U. A. Th. Brinkman., Capillary electrophoresis coupled 369 online with flame photometric detection: Determination of alkylphosphonic acids, Journal of 370 Microcolumn Separations 9 (1997) 253-259.

371 [23] M. Wheelis, Biotechnology and chemical weapons control, Pure Appl. Chem 74 (2004) $372 \quad 2247-2251$. 

ionic analytes based on covalent bond formation-a review of recent developments, Anal Bioanal Chem 386 (2006) 1201-1214.

[25] E. Climent, A. Martí, S. Royo, R. Martínez-Máñez, M. D. Marcos, F. Sancenón, J. Soto, A. M. Costero, S. Gil, M. Parra, Chromogenic Detection of Nerve Agent Mimics by Mass Transport Control at the Surface of Bifunctionalized Silica Nanoparticles, Angew. Chem. Int. Ed. 49 (2010) 5945-5948.

[26] F. Wang, H. Gu, T. M. Swanger, Carbon Nanotube/Polythiophene Chemiresistive Sensors for Chemical Warfare Agents, JACS 130 (2008) 5392-5393.

382 [27] H. J. VanTreeck, D. R. Most, B. A. Grinwald, K. A. Kupcho, A. Sen, M. D. Bonds, B. R. 383 Acharya, Quantitative detection of a simulant of organophosphonate chemical warfare agents using liquid crystals, Sensors and Actuators B 158 (2011) 104-110.

[28] Metal Oxide Semiconductor (MOS) Sensors, AppliedSensor, www.appliedsensor.com.

[29] E. Brunol, F. Berger, M. Fromm, R. Planade, Detection of dimethyl methylphosphonate (DMMP) by tin dioxide-based gas sensor: Response curve and understanding of the reactional mechanism, Sensors and Actuators B 120-1 (2006) 35-41.

[30] E. García-Breijo, V. Guarrasi, R. Masot, M. Alcañiz, C. Olguín, Odour sampling system with modifiable parameters applied to fruit classification, Journal of Food Engineering 116-2 (2013) 277-285.

[31] N. Laguarda-Miró, F. W. Ferreira, E. García-Breijo, J Ibáñez-Civera, L. Gil-Sánchez, J. Garrigues-Baixauli, Glyphosate detection by voltammetric techniques. A comparison between statistical methods and an artificial neural network, Sensors and Actuators B 171-172 (2012) 528-536.

[32] P. M. Gil, N. Laguarda-Miró, J. S. Camino, R. M. Peris, Glyphosate detection with ammonium nitrate and humic acids as potential interfering substances by pulsed voltammetry technique, Talanta 115 (2013) 702-705.

[33] I. Campos, R. Masot, M. Alcañiz, L. Gil, J. Soto, J. L. Vivancos, E. García-Breijo, R. H. Labrador, J. M. Barat, R. Martínez-Máñez, Accurate concentration determination of anions nitrate, nitrite and chloride in minced meat using a voltammetric electronic tongue, Sensors and Actuators B 149 (2010) 71-78.

403 [34] S. Royo, A. M. Costero, M. Parra, S. Gil, R. Martínez-Máñez, F. Sancenón, 404 Chromogenic, Specific Detection of the Nerve-Agent Mimic DCNP (a Tabun Mimic), Chem. Eur.

406 [35] A. M. Costero, S. Gil, M. Parra, P. M. E. Mancini, R. Martínez-Máñez, F. Sancenón, S. 
410 Cristian Olguín Pinatti has received his MS degree in Automation and Industrial Electronics 411 Engineering from the Universidad Politécnica de Valencia (UPV) in 2010 and his MS degree in 412 sensors for industrial applications from the UPV in 2012. Nowadays he is developing his Thesis 413 at the Institute of Molecular Recognition and Technological Development (IDM) of the UPV. 414 His research focuses on the electrochemical sensors, electronic noses and design of electronic 415 systems for electrochemical measurements techniques.

Nicolás Laguarda-Miró was graduated in Environmental Sciences from the Universitat 417 Politècnica de València (UPV) in 2000. He received his PhD from the same university in 2005. 418 Nicolás is nowadays a member of the Institute of Molecular Recognition and Technological 419 Development (IDM) as well as a teacher of environmental subjects in the ETSID (Escuela 420 Técnica Superior de Ingeniería del Diseño) at UPV. His main areas of interest are the 421 environmental quality control, environmental impacts assessment and technology applications 422 to these areas.

423 Lluís Pascual i Vidal graduated in Chemistry at the Universitat de València (UV) in 2011, he 424 received his Master's Degree (Master in sensors for industrial applications) at the Universitat 425 Politècnica de València (UPV) in 2012. He is a PhD Student from the Universitat Politècnica de 426 València (UPV) and his main area of interest is the development of chemical sensors and 427 probes.

428 Eduardo García-Breijo has obtained his MS degree in electronic engineering from the 429 University Polytechnic of Valencia, Spain (UPV) in 1997, and received his PhD in 2004 from the 430 University Polytechnic of Valencia. He is an assistant professor of the Electronics Engineering 431 Department of the UPV. He is a member of the Institute of Molecular Recognition and 432 Technological Development (IDM) of UPV. His main areas of interest are the development of 433 multisensors in thickfilm technology, design of electronic systems and neural networks.

434 Ramón Martínez-Mañez graduated in chemistry from the Universidad de Valencia (UV) in 435 1986, and received his PhD in 1990 at the same university. After a postdoctoral period at 436 Cambridge (UK), he joined the Department of Chemistry at the UPV. He became a full 437 professor in 2002. His main areas of interest lie in the field of chromo-fluorogenic and 438 electrochemical sensors and molecular probes for anions, cations and neutral chemical 439 species.

440 Juan Soto graduated in chemistry from the Universidad de Valencia (UV) in 1981 and received 441 his PhD in 1986 at the same University. He is currently a full professor in the Department of 442 Chemistry at the Universidad Politécnica de Valencia (UPV). His main area of interest is the 443 development of chemical chemosensors and probes, especially those based on 444 electrochemical processes. 